SMALL RNAS

\title{
Little transgressions
}

The molecular basis of transgressive segregation - which causes phenotypes of a hybrid to lie outside the range of the parental phenotypes - has been debated for decades. A new study in tomato plants indicates that transgressive phenotypes are mediated by the silencing of novel targets by small RNAs (sRNAs), which leads to genome-wide epigenetic and gene-expression changes in the hybrid.

The potential involvement of sRNAs was investigated by sequencing microRNAs and small interfering RNAs (siRNAs) in domesticated tomato seedlings and a wild relative, as well as in F1 and F2 generation hybrid progeny and in introgression lines (ILs). The aim was to identify sRNAs that were expressed at transgressively high or low levels in the hybrids, ILs, or both.

Around 1\% (153) of the detected siRNAs were transgressively expressed in the ILs and in the F2 generation. In one example, expression of siRNA from the $\mathrm{H} 06$ locus in the domesticated tomato genome was activated in trans in the hybrids, in which the H06 locus is hypermethylated and has lower levels of mRNA expression. In another example, siRNAs derived from the phenylalanine ammonialyase (PAL) locus uniquely matched several PAL ESTs in an IL line.

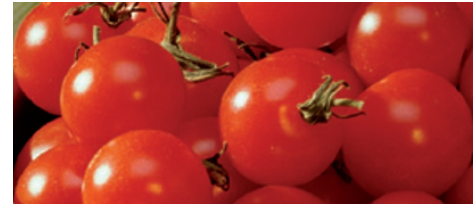

In these cases, the mechanistic explanation was consistent with an intermediate step in which a primary sRNA is converted, via the primary sRNA DNA or mRNA target, to a secondary siRNA that has a wider range of complementary targets.

This work has provided a fresh research perspective on the inheritance of non-additive phenotypes, which are important for fundamental and applied research: notably, crop breeding.

Tanita Casci

ORIGINAL RESEARCH PAPER Shivaprasad, P. V et al. Extraordinary transgressive phenotypes of hybrid tomato are influenced by epigenetics and small silencing RNAs. EMBO J. 16 Dec 2011 (doi:10.1038/emboj.2011.458) 